\title{
Posttraumatic panniculitis - Case series and literature review
}

\author{
Uwe Wollina $^{1 *}$, Birgit Heinig ${ }^{2}$, Dana Langner ${ }^{1}$, Nadine Schmidt $^{1}$ and Gesina Hansel ${ }^{1}$ \\ ${ }^{1}$ Department of Dermatology and Allergology, Academic Teaching Hospital Dresden-Friedrichstadt, Dresden, Germany \\ ${ }^{2}$ Center of Physical and Rehabilitative Medicine, Academic Teaching Hospital Dresden-Friedrichstadt, Dresden, Germany
}

\begin{abstract}
Background: Traumatic panniculitis is a rare inflammatory disease of subcutaneous adipose tissue.

Objective: We analyzed the patients' files for traumatic panniculitis in a single-center study.

Materials and methods: This is a retrospective analysis of patients seen between 04/2002 and 09/2017 at our department.

Results: We identified four adult patients, three females and one male. The age range of patients was between 23 years and 50 years. In three cases the lower legs were affected, in one case the lumbar region. A blunt trauma was reported in 100\%. Pain and subcutaneous swelling were the leading symptoms. Previous symptomatic medical treatment failed. Surgical treatment was successfully performed in three cases without relapse. Streptococcus dysgalactiae was identified in one patient.

Conclusion: Traumatic panniculitis is a rare disorder with a female predominance. Surgical treatment was successful in chronic cases, whereas medical treatment had failed.
\end{abstract}

\section{Introduction}

Panniculitides represent a heterogeneous group of inflammatory disorders of the adipose tissue [1]. Traumatic panniculitis is a rare disease of subcutaneous adipose tissue. It has been observed after blunt trauma, cold injury or surgery where the severity of adipose tissue response was not directly related to the severity of injury [2-5]. Usually it presents as a subcutaneous painful plaque, nodule or ill-defined mass with erythema. Systemic symptoms such as fever or general malaise are typically missing. In Asian patients, localized hypertrichosis may develop as a consequence of the ongoing inflammation [6-8].

We report on cases from our files in the years April 2002 to September 2017 and present a new case suggesting pyoderma and/ or lymph fistula colonized by Streptococcus dysgalactiae.

\section{Material and methods}

The files of the Department of Dermatology and Allergology, Academic Teaching Hospital Dresden, have been analyzed for the diagnosis of traumatic panniculitis from April 2002 to September 2017.

Patients were analyzed for demographics, type of trauma, clinical presentation and treatment.

\section{Results}

We identified three female patients with a confirmed diagnosis of traumatic panniculitis. All of them had at least one medical treatment before, that failed: antibiotics, anti-inflammatory non-steroidal drugs or corticosteroids.

The first patient was a 30-year-old female without any comorbidities. She presented with a tense, slowly growing pretibial mass on her left lower leg after minor blunt trauma. Imaging techniques and histopathology excluded malignancies and confirmed panniculitis ossificans. The tumor-like mass was completely removed by surgery. The defect was closed by a rotational flap [9]. During follow-up of 9 years no relapse occurred.

The second patient was a 23-year-old female patient without any comorbidities. She presented with a painful nodular mass of 25 x $6 \mathrm{~cm}$ in the right lumbar region that developed after a fall from a staircase. The lesion was excised completely and the defect could be closed by a large tissue advancement flap. Healing was uneventful. Histology confirmed adipose tissue necrosis, oily cysts and mild mixed inflammatory infiltrate as features of traumatic panniculitis [10]. Follow-up for three years remained relapse-free.

The third patient was a 50-year-old male with a blunt trauma to his right lower leg. He presented with a $7-\mathrm{cm}$ large, firm pretibial nodule. The differential diagnosis was myositis ossificans that could not be confirmed by imaging techniques. He had no co-morbidities. We discussed surgery but the patient was lost from our files.

The fourth patient is a new case. A 78-year-old woman presented with a circumscribed pretibial wound on the left lower leg that

Correspondence to: U Wollina, Department of Dermatology and Allergology and Center of Physical and Rehabilitative Medicine, Academic Teaching Hospital Dresden-Friedrichstadt, Friedrichstrasse 41, 01067, Dresden, Germany, E-mail: wollina-uw@khdf.de

Key words: Traumatic panniculitis, lower legs, blunt trauma, surgery, Streptococcus dysgalactiae

Received: September 30, 2017; Accepted: October 10, 2017; Published: October 20,2017 
developed after a blunt trauma. She suffered from chronic venous insufficiency without any previous ulcerations.

The initial clinical picture resembled pyoderma. On clinical examination, we observed a small penetrating fistulating wound with lymphorrhoea, surrounded by slightly erythematous and swollen skin measuring $4 \times 4 \mathrm{~cm}$ (Figure 1a). The lesion was painful upon pressure with a tension feeling.

An X-ray image was obtained, that demonstrated a less radiolucent soft tissue swelling. There was no bone involvement (Figure 2). Diagnostic ultrasound revealed soft tissue exudate around the fistula. A swab was taken from the wound for microbiology. Streptococcus dysgalactiae was identified.

Surgical excision of the whole lesion was performed in general anesthesia. The defect was temporarily covered by vacuum-assisted closure (Figures $1 \mathrm{~b}$ to $1 \mathrm{~d}$ ).

The tissue was subjected to histopathologic investigations. The tissue sections were stained with hematoxylin-eosin, elastic stain, Berlin-blue reaction for iron and monoclonal antibody D2-40.

We observed a chronic purulent inflammation of the subcutaneous adipose tissue with lipophagic panniculitis, necrosis of adipocytes, and erythrocyte extravasation. There were multiple oily cysts, a mixed inflammatory cellular infiltrate and sialometaplasia-like changes in eccrine sweat glands. Lymphatic vessels were unremarkable (Figure 3a-d).

After wound bed preparation, the final defect closure was realized by mesh graft transplantation. The graft take was complete and stable. Healing was unremarkable. No relapse occurred. The patient got a compression stocking, class II (Figures $3 \mathrm{a}$ and $\mathrm{b}$ ).

\section{Discussion}

Traumatic panniculitis is a rare inflammatory response of subcutaneous adipose tissue to various types of trauma. The disease can

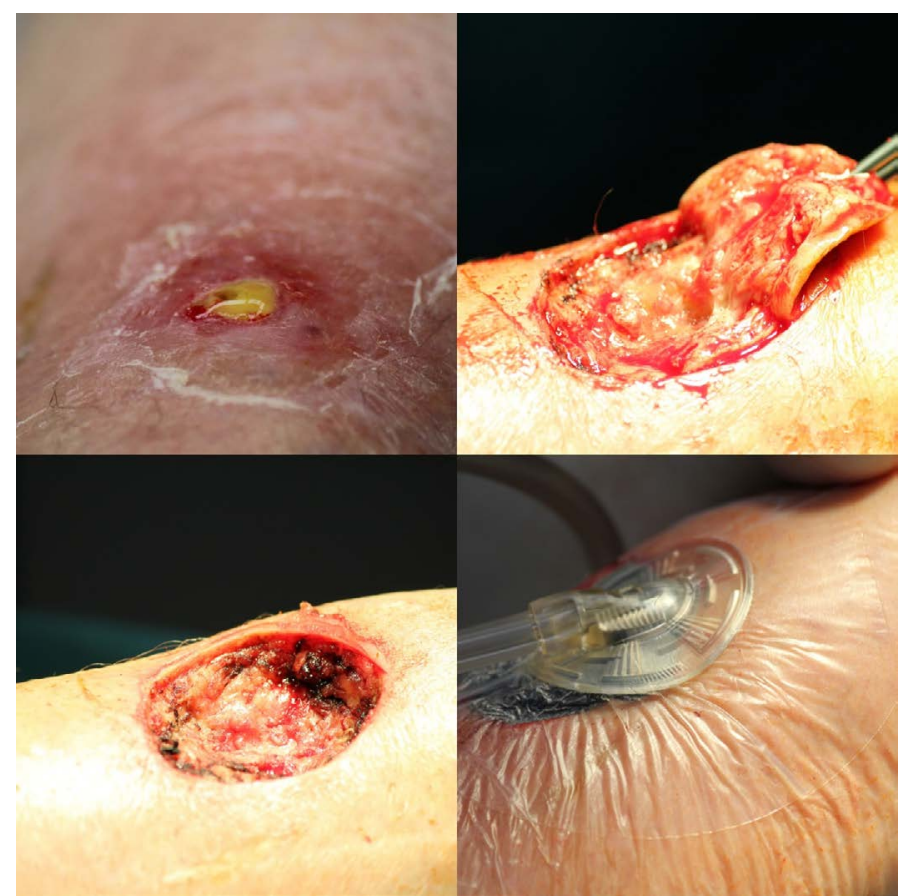

Figure 1. Traumatic panniculitis presenting as pyoderma and/or lymph fistula. (a) Initial clinical presentation (upper left). (b) Surgical removal (upper right). (c) Resulting soft tissue defect (lower left). (d) Temporary defect covering by vacuum-assisted closure (lower right).

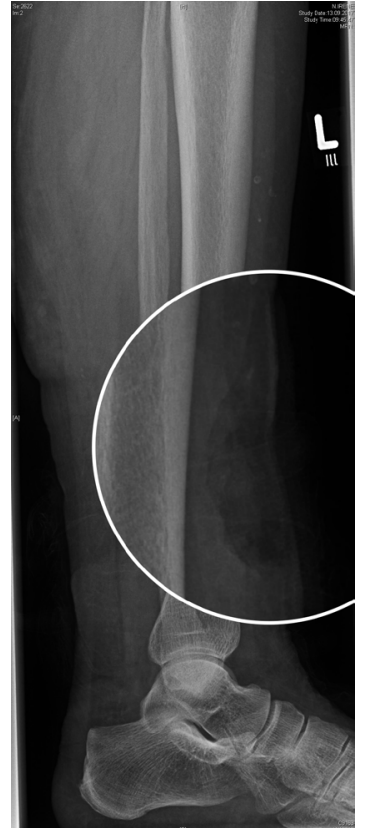

Figure 2. X-ray of the affected leg demonstrating a subcutaneous swelling but no bone involvement.

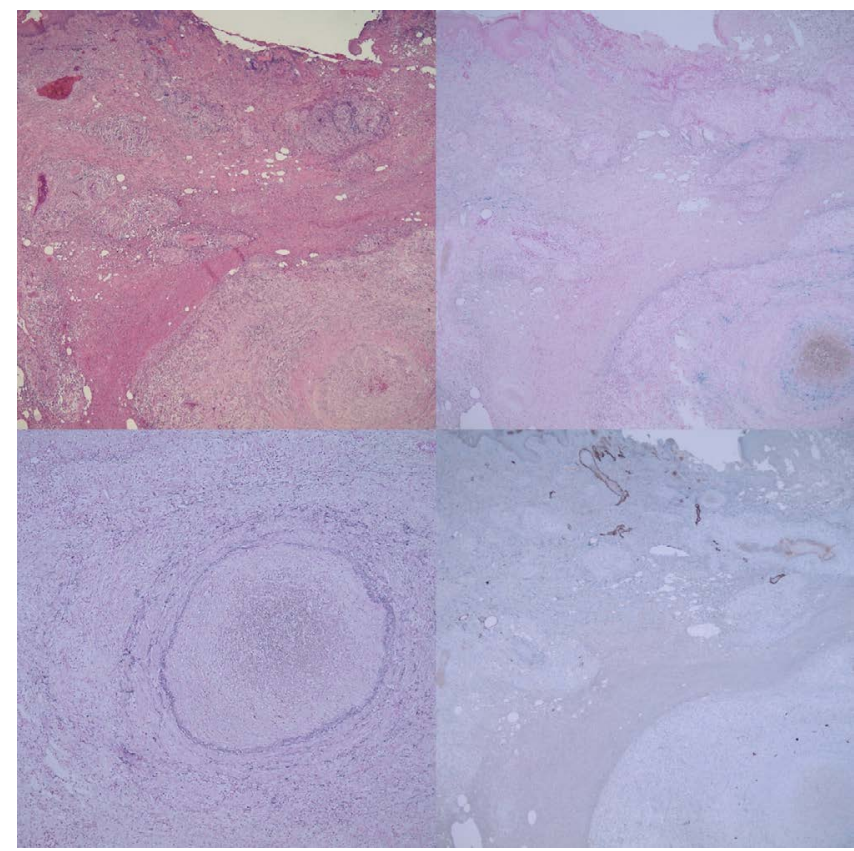

Figure 3. Histopathology of traumatic panniculitis (patient \#4). (a) Hematoxylin-eosin stain with cutaneous ulceration and lipophagic panniculitis (x 2; upper left). (b) Iron stain with extravasation of erythrocytes (x 2; upper right). (c) Detail of (b) (x 4; lower left). (d) Immunoperoxidase stain with monoclonal antibody D4-20 showing lymphatic vessels (x 2 ; lower right)

occur at any age, but in children it is self-limited. Traumatic panniculitis consists of dermal and hypodermal changes [1-3]. The histologic findings are non-specific. They include fat microcysts surrounded by histiocytes, collections of foam cells, and mixed-type of inflammatory cells. Later lesions may develop fibrosis, lipomembranous changes, pseudomembraneous changes, dystrophic calcium deposits or ectopic calcification [4, 10-12]. Sometimes, even encapsulated adipose tissue necrosis can be observed [13] (Figure 4). 


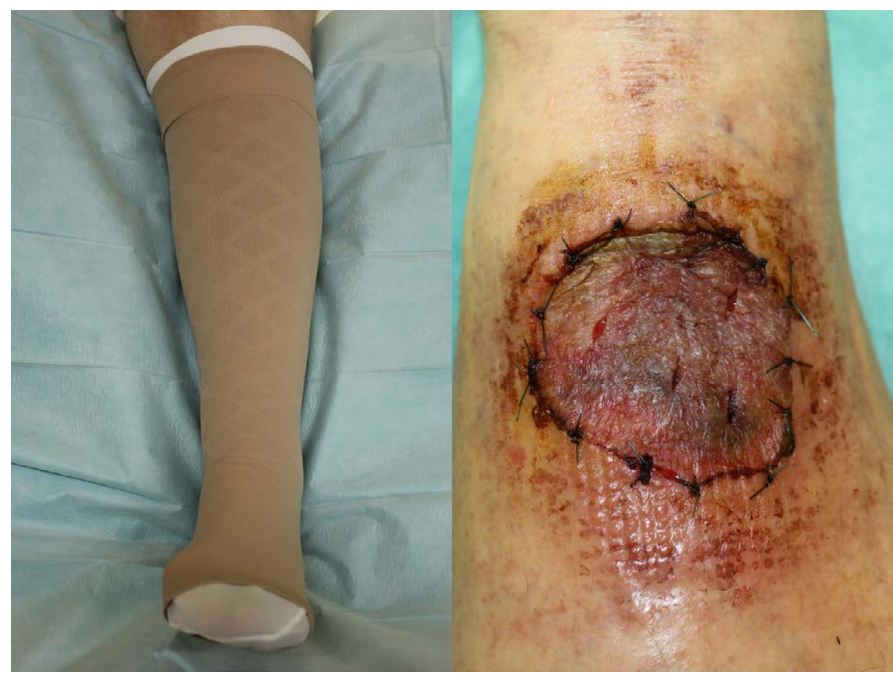

Figure 4. Outcome (patient \#4). (a) Compressions stockings with under socks (left). (b) Vital mesh graft six days after transplantation (right).

We observed only four cases in 15 years. Male to female ratio was 1:3 what is in accordance to other studies reporting a female dominance [4]. The lower legs were involved in three patients. Differential diagnoses include infections and malignancies. In three of four patients, the lesions were removed by surgery. Healing in all cases was uneventful. Medical treatments with antibiotics, corticosteroids and nonsteroidal drugs failed.

In the fourth patient, presenting initially as pyoderma, Streptococcus dysgalactiae was isolated. The species belongs to Gram-positive, group-C Streptococci, known as a commensal colonizer of the upper respiratory tract and skin forming biofilms. In recent years, however, it has been implicated in severe systemic infections and toxic shock syndrome with a high mortality rate of up to $25 \%[14,15]$. Fortunately, we did not observe systemic infection.

The recommendations for symptomatic treatment only [4] have not been purposeful in our patient series. A wait and see strategy could have set the last patient in risk for severe systemic infection. Therefore, our recommendation in case of chronic traumatic panniculitis is surgery. This has achieved complete and sustainable remissions.

\section{Reference}

1. Requena L (2007) Normal subcutaneous fat, necrosis of adipocytes and classification of the panniculitides. Semin Cutan Med Surg 26: 66-70. [Crossref]

2. Grassi S, Rosso R, Tomasini C, Pezzini C, Merlino M, et al. (2013) Post-surgical lipophagic panniculitis: a specific model of traumatic panniculitis and new histopathological findings. G Ital Dermatol Venereol 148: 435-441. [Crossref]

3. Hanif Z, Ahmad M (2006) Subcutaneous fat necrosis presenting as a large mass. Eur J Emerg Med 13: 106-107. [Crossref]

4. Moreno A, Marcoval J, Peyri J (2008) Traumatic panniculitis. Dermatol Clin 26: 481 483, vii. [Crossref]

5. Lipke MM, Cutlan JE, Smith AC1 (2015) Cold panniculitis: delayed onset in an adult Cutis 95: 21-24. [Crossref]

6. Lee DJ, Kim YC (2011) Traumatic panniculitis with hypertrichosis. Eur J Dermatol 21: 258-259. [Crossref]

7. Lee JH, Jung KE, Kim HS, Kim HO, Park YM, et al. (2013) Traumatic panniculitis with localized hypertrichosis: two new cases and considerations. J Dermatol 40: 13941. [Crossref]

8. Oh CC, Tan KB, Thirumoorthy T, Pang SM, Lee HY (2014) Traumatic panniculitis in a Chinese woman. Skinmed 12: 127-128. [Crossref]

9. Wollina U, Koch A, Schönlebe J, Witzigmann H, Kittner T (2009) Panniculitis ossificans of the lower leg. Int J Low Extrem Wounds 8: 165-168. [Crossref]

10. Wollina U (2010) Posttraumatic painful subcutaneous oily cysts. Kosmet Med 35 128-129.

11. Diaz-Cascajo C, Borghi S (2002) Subcutaneous pseudomembranous fat necrosis: new observations. J Cutan Pathol 29: 5-10. [Crossref]

12. Fernandez-LE, Peñá PC, Garcia SJ, Unamuno P (2002) Membranous fat necrosis: non-specific histological finding. Eur J Dermatol 12: 82-84.

13. Santos JJ, Coto P, Galache C, Sánchez J, Soto DJ (2007) Encapsulated fat necrosis: a form of traumatic panniculitis. J Eur Acad Dermatol Venereol 21: 405-406. [Crossref]

14. Ashchyan HJ, Brown KK (2017) Cutaneous eruption associated with Streptococcus dysgalactiae (group C Streptococcus) bacteremia. JAAD Case Rep 3:373-375. [Crossref]

15. Bradley SF, Gordon JJ, Baumgartner DD, Marasco WA, Kauffman CA (1991) Group C streptococcal bacteremia: analysis of 88 cases. Rev Infect Dis 13:270-280. [Crossref]

Copyright: $\odot 2017$ Wollina U. This is an open-access article distributed under the terms of the Creative Commons Attribution License, which permits unrestricted use, distribution, and reproduction in any medium, provided the original author and source are credited. 